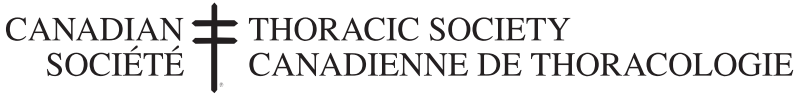

\section{The good COPD guidelines}

\section{De bonnes lignes directrices sur la MPOC}

$\mathrm{C}$ hronic obstructive pulmonary disease (COPD) is becoming an increasing problem for health care workers. The World Health Organization predicts that in the year 2020, this disease will be the fifth most prevalent disease worldwide, up from 12 th place, and it will be the third most common cause of death, up from sixth place in 1997 (1). Hospitalization and mortality rates for COPD continue to rise in Canada (2). Therefore, the burden of COPD on Canadians, the health care system and physicians is obvious.

The release of the new Canadian Census Guidelines (3) is both timely and appropriate. The work involved a tremendous amount of man-hours by the Canadian Thoracic Society COPD Committee and its chair, Denis O'Donnell. The result is the most up-to-date guidelines in the world on this subject, with some unique features. They chronicled a tremendous amount of recent research in a field in which Canadians have made major contributions in almost all areas. For a number of years, Canadians have been and continue to be involved in research concerning COPD. This has resulted in huge Canadian contributions regarding COPD in the mechanisms of dyspnea, the pathology of the illness, the lung mechanics with its abnormal function, the systemic effects of this devastating disease and the treatment of patients. All of this is documented in the excellent bibliography, which substantiates the data presented. The immensity of this task should be appreciated because of the great amount of work put into it by numerous health care workers, which involved broad consultation with many individuals to make it a truly composite document.

The document clearly indicates that for patients with chronic obstructive lung disease - who, for so long, we considered to be beyond help - there is much that can be done to improve their quality of life besides smoking cessation. It highlights the importance of educating the patient and gives justifiable importance to pulmonary rehabilitation, which may be the most useful therapeutic modality in improving the lives of obstructive lung disease sufferers. COPD has been documented to be a systemic disease with muscle wasting, weakness and nutritional problems with its necessary effects on these individuals. These facts highlight the importance of exercise and education in helping our patients.

In addition, the manuscript clearly points out the significance of repeated, acute exacerbations of COPD, which have important implications for patients $(4,5)$. It gives practical management tips for such exacerbations and the rationale behind aggressive treatment for them. It indicates the roles of bronchodilators, corticosteroid therapy, antibiotic use and noninvasive mechanical ventilation.

The paper is a very useful document for family physicians in that it gives a simple classification for the severity of COPD, relating it to symptom assessment. There is clear evidence that
T a maladie pulmonaire obstructive chronique L(MPOC) est un problème croissant pour les dispensateurs de soins. L'Organisation mondiale de la santé prévoit qu'en 2020, la prévalence de cette maladie sera en cinquième place dans le monde, par rapport à une douzième place, et elle sera la troisième cause de décès en importance, par rapport à une sixième place en 1997 (1). Les taux d'hospitalisation et de mortalité de la MPOC continuent d'être en hausse au Canada (2\%). Par conséquent, le fardeau de la MPOC est évident sur les Canadiens, le système de santé et les médecins.

La publication des nouvelles lignes directrices canadiennes du recensement (3) est à la fois d'actualité et pertinente. Le travail a exigé un nombre très élevé d'heures-personnes au comité de la MPOC de la Société canadienne de thoracologie et à son président, Dennis O'Donnell. Le résultat? Les lignes directrices les plus à jour au monde sur le sujet, et qui comportent des caractéristiques uniques. Elles colligent un nombre important de recherches récentes dans un domaine auquel les Canadiens ont contribué de manière significative dans presque tous les secteurs. Depuis plusieurs années, les Canadiens participent à la recherche sur la MPOC. Ces recherches ont donné lieu à d'énormes contributions canadiennes quant à l'apport de la MPOC dans le mécanisme de la dyspnée, la pathologie de la maladie, les mécaniques pulmonaires et leurs fonctions anormales, les effets systémiques de cette maladie dévastatrice et le traitement des patients. Toutes ces recherches sont documentées dans l'excellente bibliographie qui corrobore les données présentées. On peut se rendre compte de l'immensité de la tâche par le travail acharné d'innombrables dispensateurs de soins, lequel a exigé une vaste consultation auprès de nombreuses personnes afin que le document soit véritablement composite.

Le document indique clairement que, chez les patients atteints d'une MPOC, pour qui on a longtemps pensé qu'il n'y avait rien à faire, il y a, au contraire, beaucoup à faire pour améliorer la qualité de vie, en plus de l'abandon du tabac. Ce constat souligne l'importance d'éduquer le patient et justifie la réadaptation pulmonaire, qui est peut-être la modalité thérapeutique la plus utile pour améliorer la vie des personnes atteintes d'une MPOC. Il est documenté que la MPOC est une maladie systémique qui s'accompagne de perte musculaire, de faiblesse et de troubles nutritionnels, lesquels ont des effets indéniables sur les personnes atteintes. Ces faits démontrent l'importance de l'exercice et de l'éducation pour aider les patients.

De plus, le manuscrit expose en toute clarté la signification d'exacerbations aiguës et répétées de la MPOC, qui ont des répercussions importantes pour les patients $(4,5)$. Il contient des conseils pratiques de prise en charge dans le cas de ces exacerbations et la raison d'être de leur traitement énergique. Il indique le rôle des bronchodilatateurs, de la corticoïdothérapie, de l'usage des antibiotiques et de la ventilation mécanique non envahissante.

Larticle est un document très utile pour les médecins de famille, car il offre une classification simple de la gravité de la 
dyspnea may be a more important predictor than spirometry in determining survival (6). This classification is based on the well-known British Medical Research Council's dyspnea scale, which has been in use for years, and enough research has been done to make it a sensible classification of severity, which has major implications for aggressiveness of treatment. Such a table could easily be accessible on a doctor's desk for day-to-day use. The authors give practical tips on escalating therapy, ie, when and how to use which bronchodilators, etc. They talk about the appropriate role of corticosteroids and when to use this class of drug. There are useful tips on when such patients should be referred to a specialist. Finally, unlike other guidelines, they discuss the importance of end-of-life issues. It encourages dialogue by primary care physicians early on so as to have patients participate in their care, as well as to have physicians reassure them that we can and will address their concerns.

This excellent and practical document has much to offer the practicing physician, although it appears long - some 22 pages without references (there are many tables and figures, making this a readable and useable document). In addition, the authors have provided a three-page summary containing the main messages. However, I recommend reading the executive summary in its entirety for those involved in the care of COPD patients.

In summary, the new "Canadian Thoracic Society Recommendations for Management of Chronic Obstructive Pulmonary Disease" is a practical outline of the management of a disease no longer considered hopeless. Many advances are documented that can considerably improve quality of life in these patients. In their efforts, they have clearly documented the major contributions that Canadians have made in all aspects of this very difficult disease. The major work to come is to implement this excellent work so that those patients with this severe malady receive significant benefit. The COPD committee has charged Dr Paul Hernandez with this task, and his committee has been very active in the past six months in developing a plan for implementation over the next few years.

Dennis Bowie MD President, Canadian Thoracic Society

\section{REFERENCES}

1. Lopez AD, Murray CC. The global burden of disease, 1990-2020. Nat Med 1998;4:1241-3.

2. Centre for Chronic Disease Prevention and Control, Health Canada, Canadian Institute for Health Information, Editorial Board. Respiratory Disease in Canada. Ottawa: Health Canada, 2003.

3. O'Donnell DE, Aaron S, Bourbeau J, et al. Canadian Thoracic Society. Canadian Thoracic Society recommendations for management of chronic obstructive pulmonary disease -2003 . Can Respir J 2003;10(Suppl A):1A-33A.

4. Seemungal TA, Donaldson GC, Paul EA, Bestall JC, Jeffries DJ, Wedzicha JA. Effect of exacerbation on quality of life in patients with chronic obstructive pulmonary disease. Am J Respir Crit Care Med 1998;157:1418-22.

5. Kanner RE, Anthonisen NR, Connet JE. Lower respiratory illness promotes $\mathrm{FEV}_{1}$ decline in current smokers but not ex-smokers with mild chronic obstructive pulmonary disease. Am J Respir Crit Care Med 2001;164:358-64.

6. Nishimura K, Izumi T, Tsukino M, Oga T. Dyspnea is a better predictor of 5 -year survival than airway obstruction in patients with COPD. Chest 2002;121:1434-40.
MPOC par rapport à l'évaluation des symptômes. Il est clairement démontré que la dyspnée est un meilleur prédicteur de survie que la spirométrie (6). Ce classement se fonde sur l'échelle bien connue de dyspnée du conseil britannique de recherche médicale, utilisée depuis de nombreuses années, et des recherches suffisantes ont été effectuées pour en faire un mode rationnel de classement de la gravité, ce qui a une grande importance quant au choix plus ou moins énergique du traitement. Un tel tableau pourrait facilement être placé sur le bureau d'un médecin pour son usage quotidien. Les auteurs donnent des conseils pratiques sur le traitement progressif, c'est à dire quand et comment utiliser quel bronchodilatateur, etc. Ils traitent du rôle pertinent des corticoïdes et des situations dans lesquelles utiliser cette classe de médicament. Ils proposent des conseils utiles sur le moment d'orienter le patient vers un spécialiste. Enfin, contrairement aux autres lignes directrices, ils expliquent l'importance des questions de fin de vie. L'article incite le médecin de premier recours à ouvrir rapidement le dialogue pour que les patients participent à leurs soins et à rassurer les patients en leur garantissant que nous pouvons et que nous allons réagir à leurs préoccupations.

Ce document, pratique et excellent, a beaucoup à offrir au médecin traitant, même s'il semble long, avec ses quelque 22 pages, sans compter les références (en raison des nombreux tableaux et des nombreuses figures, il est tout de même utile et facile à lire). De plus, les auteurs ont fourni un sommaire de trois pages qui renferme les principaux messages. Je recommande toutefois à ceux qui participent aux soins des patients atteints de MPOC de lire l'ensemble du résumé.

Bref, les nouvelles Recommandations de la Société canadienne de thoracologie au sujet de la prise en charge de la maladie pulmonaire obstructive chronique sont un exposé pratique de la prise en charge d'une maladie qu'on ne considère plus comme sans espoir. De nombreux progrès documentés peuvent améliorer considérablement la qualité de vie de ces patients. Dans leurs efforts, les auteurs ont clairement démontré les importantes contributions des Canadiens à tous les aspects de cette maladie très complexe. Les principaux travaux à venir consisteront à implanter ces recommandations pour que les patients atteints de cette maladie grave en tirent des bénéfices marqués. Le comité de la MPOC a confié cette tâche au docteur Paul Hernandez, et depuis six mois, son comité s'active à élaborer un plan d'implantation réparti sur les quelques prochaines années.

Dennis Bowie MD

Président, Société canadienne de thoracologie

\section{RÉFÉRENCES}

1. Lopez AD, Murray CC. The global burden of disease, 1990-2020. Nat Med 1998;4:1241-3.

2. Centre de prévention et de contrôle des maladies chroniques, Santé Canada, Institut canadien d'information sur la santé, comité de rédaction. Les maladies respiratoires au Canada. Ottawa : Santé Canada, 2003.

3. O'Donnell DE, Aaron S, Bourbeau J, et al. Canadian Thoracic Society. Canadian Thoracic Society recommendations for management of chronic obstructive pulmonary disease - 2003. Can Respir J 2003;10(Suppl A):1A-33A.

4. Seemungal TA, Donaldson GC, Paul EA, Bestall JC, Jeffries DJ, Wedzicha JA. Effect of exacerbation on quality of life in patients with chronic obstructive pulmonary disease. Am J Respir Crit Care Med 1998;157:1418-22.

5. Kanner RE, Anthonisen NR, Connet JE. Lower respiratory illness promotes $\mathrm{FEV}_{1}$ decline in current smokers but not ex-smokers with mild chronic obstructive pulmonary disease. Am J Respir Crit Care Med 2001;164:358-64.

6. Nishimura K, Izumi T, Tsukino M, Oga T. Dyspnea is a better predictor of 5-year survival than airway obstruction in patients with COPD. Chest 2002;121:1434-40. 


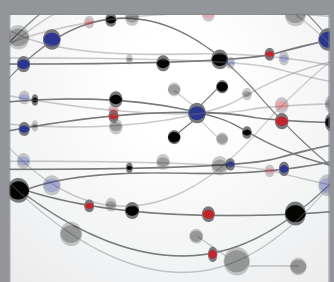

The Scientific World Journal
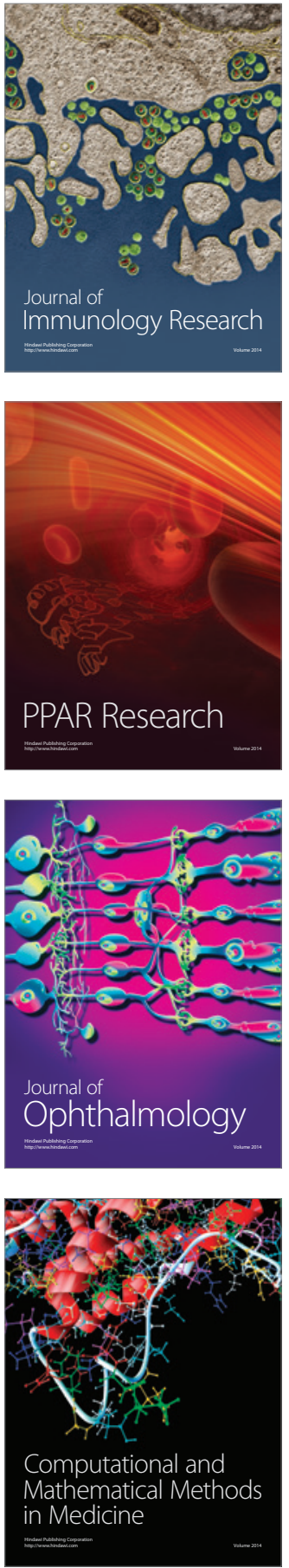

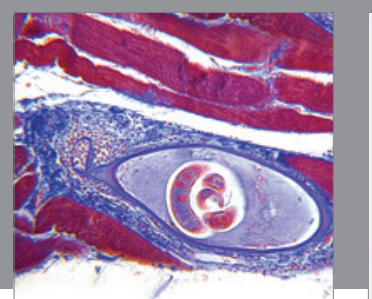

Gastroenterology Research and Practice

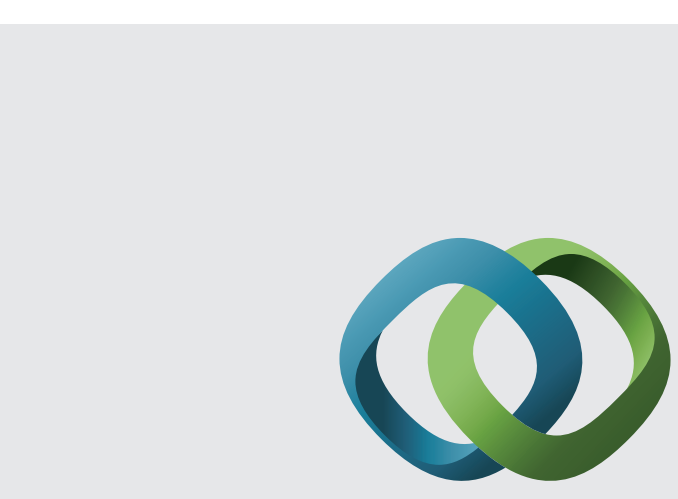

\section{Hindawi}

Submit your manuscripts at

http://www.hindawi.com
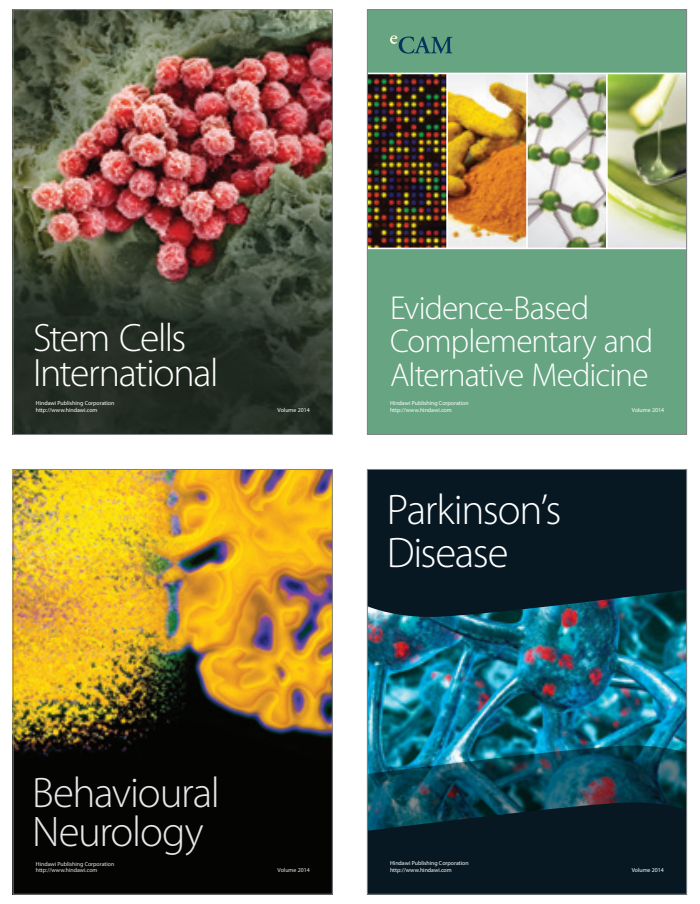
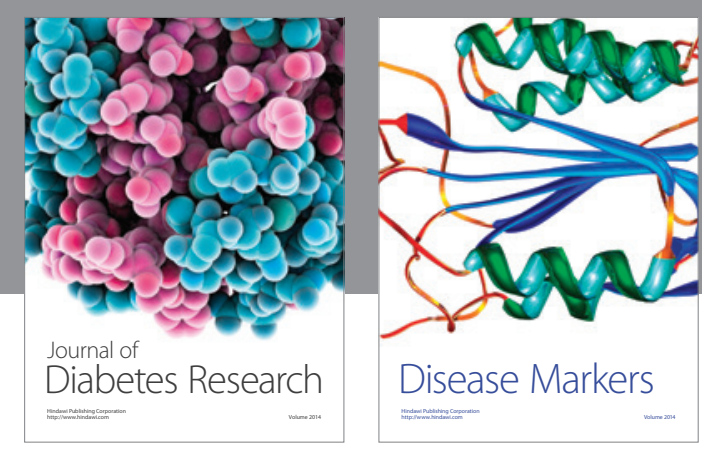

Disease Markers
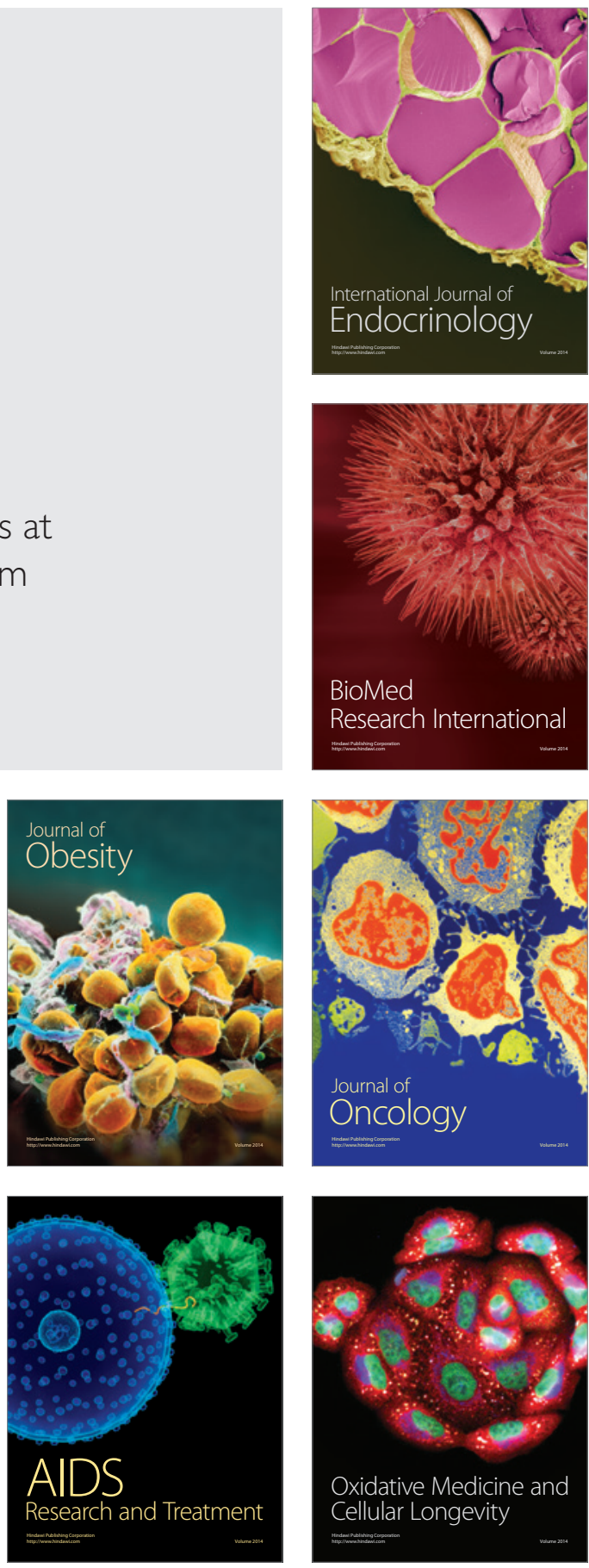